
\title{
25 Research Square \\ Low Serum Iron Status Is Associated With Low Incidence of Coronary Artery Disease In Women
}

Shuren Guo ( $\sim$ guoshuren@126.com )

The First Affiliated Hospital of Zhengzhou University

Xiaohuan Mao

People's Hospital of Zhengzhou University

Xiaohua Li

The First Affiliated Hospital of Zhengzhou University

Huan Ouyang

ShenQiu People's Hospital

\section{Research Article}

Keywords: Serum iron, ferritin, UIBC, TIBC, CAD, women

Posted Date: January 6th, 2022

DOI: https://doi.org/10.21203/rs.3.rs-1175662/v1

License: (a) (i) This work is licensed under a Creative Commons Attribution 4.0 International License. Read Full License 


\section{Abstract}

Background: Disorders of iron metabolism has been implicated in cardiovascular disease. However, the association of serum ferritin and coronary artery disease (CAD) remains inconsistent. Here, we investigated the associations of serum iron metabolism with the incidence of CAD, the severity of coronary artery stenosis, metabolic biomarkers, and 1-year restenosis after coronary artery revascularization.

Methods: A total of 643 CAD patients and 643 healthy controls were enrolled to assess the associations of serum iron status with the presence of CAD, the severity of CAD, and 1-year rehospitalization after revasculation. Serum iron metabolism and other metabolic markers were measured in all subjects. All statistical analyses were analyzed using SPSS22.0 software and STATA statistical package.

Results: Serum level of iron metabolism markers, including serum iron, ferritin, unsaturated transferrin iron binding capacity (UIBC), Total iron binding capacity (TIBC) levels, in CAD groups was significantly higher than the control group $(P<0.001)$. UIBC and TIBC were negatively correlated with ferritin in both sexes. Serum level of iron $(\mathrm{OR}=0.806,95 \% \mathrm{Cl}(0.687-0.944), \mathrm{UIBC}(\mathrm{OR}=0.919,95 \% \mathrm{Cl}(0.852-0.992)$, and TIBC (OR=0.864, 95\% Cl (0.787-0.95) were found to have a protective role for CAD in women ( $P<0.05$, Table 3$)$. The $O R$ for ferritin was significant in the both sexes $(\mathrm{OR}=1.029,95 \% \mathrm{Cl}(1.002-1.058)$ in men, $\mathrm{OR}=1.02,95 \% \mathrm{Cl}(1.005-1.034)$ in women, $\mathrm{P}<0.05)$.

Conclusion: Low Serum level of iron, UIBC, TIBC and ferritin levels were found to have a protective role for CAD in women, but not in men. Elevated serum ferritin is independently and positively associated with CAD in men and women.

\section{Introduction}

Iron plays a role in several fundamental biological processes such as erythropoiesis and cell metabolism. Iron status is a modifiable feature associated with cardiovascular disease. Iron metabolism disorders, either deficiency or overload, were associated with increased cardiovascular morbidity and mortality [1,2]. The increase of iron stores was also positively correlated with the risk factors of cardiovascular disease, such as the risk of metabolic syndrome [3], insulin resistance (IR) [4, 5] and new-onset type 2 diabetes mellitus [6]. The role of body iron indices has been reviewed in the pathogenesis of coronary artery disease (CAD) [7-11]. It has been suggested iron plays an important role in the pathogenesis of atherosclerosis by catalyzing the atherogenic modification of low density lipoprotein (LDL) [10, 12-16]. Some studies supported the iron hypothesis [4, 17-21], some found no supporting evidence, and the results of others contradicted the hypothesis [22-26].

Although iron status was implicated in cardiovascular disease, the relationship between iron states and CAD has long been a controversial topic in the literature. Because of the inconsistent results of the association between body iron stores and CAD risk, we conducted a case-control study enrolled a total of 643 CAD patients and 643 healthy controls to analyze the associations of serum iron levels with the presence of $C A D$, the severity of coronary artery stenosis, and 1-year rehospitalization after revasculation.

\section{Subjects And Methods \\ Study population}


The control group consisted of 643 (male/female 381/262) healthy persons without cardiovascular disease via physical examination and electrocardiogram. The controls were frequency-matched to the cases on age and sex. The CAD group consisted of 643 patients, 349 men and 294 women. All CAD patients underwent physical examination and review of their medical history. Patients were excluded if they 1) had heart failure, acute myocardial infarction, coronary bypass surgery or angioplasty, coronary spasm, or myocardial bridge; 2) had cardiac diseases such as cardiomyopathy, valvular or congenital heart disease, arrhythmia; 3) patients who had malignant tumors, acute or chronic infection, iron deficiency anemia, digestive system disease, fever, infection, connective tissue disease, autoimmune disease; 4) patients whose Blood pressure $\geq 180 / 110 \mathrm{mmHg}$ after taking standard antihypertensive drugs, severe hepatic or renal dysfunction; 5) A history of major surgical trauma, pregnancy, mental illness, or any other cause of active blood loss within 2 weeks. The Zhengzhou University ethics Committee approved this study (The approval number 2020-KY-172). All the investigations were performed in accordance with the principles of the Declaration of Helsinki. Informed consent was obtained from all participants or from a parent or legal guardian if participants are under 16.

\section{Methods}

All subjects underwent anthropometrical evaluation with measurements of weight, height, and body mass index (BMI). Laboratory determinations: All blood samples were obtained after overnight fasting. Fasting plasma glucose (FPG), low-density lipoprotein cholesterol (LDL-C), high-density lipoprotein cholesterol (HDL-C), total cholesterol (TC), triglycerides (TG), high sensitive C-reactive protein (hs-CRP), iron metabolism biomarkers, liver function and renal function markers were measured on a Cobas 8000 Analyzer (Roche Diagnostics, Germany) using the Original Roche Reagent following the manufacturer's manual.

Plasma glucose was measured by the hexokinase method; TG, TC, LDL-C and HDL-C, Alanine aminotransferase (ALT) and aspartate aminotransferase (AST), creatinine, urea nitrogen (UREA), uric acid (UA) concentrations were evaluated by enzymatic methods. Hemoglobin A1c (HbA1c) was assayed by high-performance liquid chromatography on a Primer-Premier Hemoglobin Testing System (reagents were supplied by Primus). hs-CRP, homocysteine (Hcy), Apolipoprotein A (ApoA), Apolipoprotein B (ApoB) and serum ferritin levels were measured using an immune-turbid metric assay. The serum iron was measured with a colorimetric method; while the levels of unsaturated transferrin iron binding capacity (UIBC) was measured using a colorimetric test. Total iron binding capacity (TIBC) equaled the serum iron levels plus serum UIBC levels. Finally, estimated glomerular filtration rate (eGFR) was computed by using the chronic kidney disease epidemiology (CKD-EPI) collaboration equation [27]. Iron deficiency was defined as: ferritin $<100 \mathrm{ng} / \mathrm{mL}$ or $100-300 \mathrm{ng} / \mathrm{mL}$ with transferrin saturation $(\mathrm{Tfs})<20 \%$ [28]. Ferritin were categorized in low (<30ng/mL; women CAD/Control $n=6 / 40$, male CAD/Control $n=6 / 8)$, normal (30200ng/mL in women; CAD/Control $\mathrm{n}=168 / 197$, and 30-300ng/mL in men; CAD/Control $\mathrm{n}=204 / 304$ ), and high (>200ng/mL in women, CAD/Control $n=120 / 25$, and >300ng/mL in men; CAD/Control $n=139 / 69$ ) ferritin levels [21, 29-31]. Selective cardioangiography (CAG) was performed in all patients using the standard Judkins technique. The localization of coronary artery disease and the rate of lumen stenosis were determined by CAG.

\section{Statistical analysis}

Continuous variables were expressed as mean \pm standard deviation (normally distributed data). Categorical variables are expressed as the frequency and its percentage. Continuous variables were analyzed using Student's ttest in normally distributed data, and Mann-Whitney test in non-normally distributed data. Chi-squared test was 
utilized for categorical variables. The association between continuous variables was assessed by Pearson correlation. The association of iron metabolism biomarkers with CAD was analyzed by logistic regression models with three progressive degrees of adjustment. Model 1 was a crude model without any confounders; model 2 was adjusted for age and cardiovascular risk factors including smoking habit, alcohol drinking habit, body mass index (BMI), hypertension, and dyslipidemia; model 3 was additionally adjusted for laboratory tests including HbA1C, ALT, AST, TC, TG, hs-CRP, eGFR. To avoid collinearity, we did not include iron and transferrin saturation into the multiple liner regression models because these two variables lied into the same causal pathway between ferritin and CAD. All the statistical analyses were executed using Statistical Package for Social Science (SPSS, version 22.0) and STATA statistical package (version 13, Texas, USA).

\section{Results}

\section{Clinical characteristics}

Table 1 showed the general characteristics of the study groups according to the sexual groups. There was no significant difference in age and diastolic blood pressure (DBP) between CAD group and controls. Overall, CAD patients were more likely to be smokers, alcohol drinkers, obese, hypertensive, dyslipidemia and hyperglycemia. Comparing to the controls, individuals in the male CAD group showed significantly lower plasma concentrations of Total proteins (TP), Albumin (ALB), and ApoB, Tfs serum iron levels, systolic blood pressure (SBP) and body mass index (BMI). The plasma concentrations of ferritin, UIBC, HbA1c, FBP, hs-CRP, liver function and renal function were significantly higher in the CAD group compared to the control in men $(P<0.05)$ (Table 1, Figure 1). Similar difference was found in the female group (Table 1, Figure 1). Additionally, significant differences were found in the diabetes management styles between male and female patients with CAD (Table 1). 
Table 1

Clinical characteristics of patients and controls

\begin{tabular}{|c|c|c|c|c|c|c|}
\hline & male & & & female & & \\
\hline & $\begin{array}{l}\text { Control } \\
(n=381)\end{array}$ & $\operatorname{CAD}(n=349)$ & $P$ & $\begin{array}{l}\text { Control } \\
(n=401)\end{array}$ & $\operatorname{CAD}(n=294)$ & $P$ \\
\hline Smoking n (\%) & $179(46.98)$ & $244(70)$ & $<0.001$ & $154(38.40)$ & $115(39.25)$ & 0.82 \\
\hline Drinking n (\%) & $174(45.67)$ & $128(36.68)$ & 0.014 & $45(11.22)$ & $25(8.53)$ & 0.24 \\
\hline age(years) & $53.05 \pm 10.75$ & $58.56 \pm 10.82$ & 0.052 & $49.38 \pm 12.09$ & $61.9 \pm 10.48$ & 0.128 \\
\hline $\mathrm{BMI}(\mathrm{kg} / \mathrm{m} 2)$ & $22.99 \pm 2.46$ & $24.23 \pm 2.55$ & 0.095 & $22.98 \pm 1.87$ & $24.18 \pm 2.68$ & $<0.001$ \\
\hline $\mathrm{SBP}(\mathrm{mmHg})$ & $120.32 \pm 9.28$ & $127.92 \pm 17.07$ & $<0.001$ & $120.79 \pm 9.73$ & $130.94 \pm 17.7$ & $<0.001$ \\
\hline $\mathrm{DBP}(\mathrm{mmHg})$ & $74.84 \pm 9.04$ & $78.76 \pm 11.14$ & 0.426 & $74.38 \pm 9.02$ & $78.45 \pm 11.8$ & 0.457 \\
\hline HR(heart ratio) & $70.21 \pm 5.08$ & $77.45 \pm 10.47$ & $<0.001$ & $69.78 \pm 4.96$ & $77.66 \pm 10.64$ & $<0.001$ \\
\hline $\begin{array}{l}\text { Smoking } \\
\text { status (\%) }\end{array}$ & $60(15.75)$ & 118(33.81) & $<0.001$ & 48(11.97) & $94(31.97)$ & $<0.001$ \\
\hline Drinker (\%) & $56(14.7)$ & $101(28.94)$ & $<0.001$ & $45(11.22)$ & $87(29.59)$ & $<0.001$ \\
\hline HbA1c (\%) & $5.4 \pm 0.45$ & $6.09 \pm 1.21$ & $<0.001$ & $5.46 \pm 0.47$ & $6.29 \pm 1.36$ & $<0.001$ \\
\hline $\mathrm{FPG}(\mathrm{mmol} / \mathrm{L})$ & $5.06 \pm 0.6$ & $5.33 \pm 1.79$ & $<0.001$ & $5.04 \pm 0.7$ & $5.58 \pm 1.88$ & $<0.001$ \\
\hline $\operatorname{ALT}(\mathrm{U} / \mathrm{L})$ & $24.31 \pm 17.02$ & $42.57 \pm 125.79$ & 0.004 & $17.06 \pm 9.6$ & $40.54 \pm 225.86$ & 0.042 \\
\hline AST(U/L) & $22.22 \pm 11.71$ & $32.17 \pm 38.03$ & $<0.001$ & $19.95 \pm 7.52$ & $54.75 \pm 417.38$ & 0.056 \\
\hline TBIL $(\mu \mathrm{mol} / \mathrm{L})$ & $12.99 \pm 5.27$ & $12.19 \pm 8.66$ & 0.008 & $10.19 \pm 4.77$ & $10.61 \pm 14.93$ & 0.19 \\
\hline BILD $(\mu \mathrm{mol} / \mathrm{L})$ & $5.53 \pm 1.85$ & $5.4 \pm 4.34$ & 0.002 & $4.68 \pm 1.77$ & $5 \pm 12.31$ & 0.156 \\
\hline $\mathrm{GGT}(\mathrm{U} / \mathrm{L})$ & $38.69 \pm 56.96$ & $36.9 \pm 33.61$ & 0.466 & $30.53 \pm 19.61$ & $32.33 \pm 43.83$ & 0.251 \\
\hline $\mathrm{TP}(\mathrm{g} / \mathrm{L})$ & $72.86 \pm 4.09$ & $65.46 \pm 5.41$ & $<0.001$ & $74.1 \pm 3.81$ & $66.68 \pm 5.62$ & $<0.001$ \\
\hline $\operatorname{ALB}(\mathrm{g} / \mathrm{L})$ & $47.32 \pm 2.82$ & $40.94 \pm 4.02$ & $<0.001$ & $46.78 \pm 2.39$ & $40.93 \pm 4.34$ & $<0.001$ \\
\hline $\operatorname{ALP}(\mathrm{U} / \mathrm{L})$ & $70.58 \pm 17.4$ & $72.22 \pm 21.25$ & $<0.001$ & $69.72 \pm 22.4$ & $79.45 \pm 39.34$ & 0.001 \\
\hline $\mathrm{TC}(\mathrm{mmol} / \mathrm{L})$ & $4.64 \pm 0.99$ & $3.41 \pm 0.88$ & 0.574 & $4.64 \pm 0.95$ & $3.9 \pm 1.09$ & 0.126 \\
\hline $\mathrm{TG}(\mathrm{mmol} / \mathrm{L})$ & $1.74 \pm 1.07$ & $1.49 \pm 1.03$ & 0.052 & $1.29 \pm 0.7$ & $1.58 \pm 1.25$ & 0.046 \\
\hline $\mathrm{HDL}(\mathrm{mmol} / \mathrm{L})$ & $1.2 \pm 0.29$ & $0.99 \pm 0.24$ & 0.084 & $1.43 \pm 0.31$ & $1.12 \pm 0.31$ & 0.983 \\
\hline $\mathrm{LDL}(\mathrm{mmol} / \mathrm{L})$ & $2.91 \pm 0.87$ & $1.95 \pm 0.79$ & 0.653 & $2.83 \pm 0.84$ & $2.23 \pm 0.88$ & 0.338 \\
\hline APOA (g/L) & $1.37 \pm 0.26$ & $1.11 \pm 0.26$ & 0.958 & $1.43 \pm 0.25$ & $1.17 \pm 0.26$ & 0.018 \\
\hline APOB $(g / L)$ & $0.86 \pm 0.27$ & $0.84 \pm 0.36$ & 0.028 & $0.84 \pm 0.21$ & $0.94 \pm 0.42$ & 0.002 \\
\hline Lpa $(g / L)$ & $0.16 \pm 0.17$ & $0.33 \pm 1.78$ & 0.216 & $0.16 \pm 0.17$ & $0.25 \pm 0.27$ & $<0.001$ \\
\hline HCY (umol/L) & $13.59 \pm 9.17$ & $15.58 \pm 9.08$ & 0.698 & $13.31 \pm 7.29$ & $15.69 \pm 8.39$ & 0.732 \\
\hline
\end{tabular}




\begin{tabular}{|c|c|c|c|c|c|c|}
\hline \multicolumn{4}{|c|}{ male } & \multicolumn{3}{|l|}{ female } \\
\hline hsCRP (mg/L) & $1.61 \pm 1.61$ & $5.68 \pm 14.05$ & 0.004 & $1.09 \pm 0.88$ & $8.84 \pm 25.41$ & 0.003 \\
\hline $\begin{array}{l}\text { CREA } \\
(\mu \mathrm{mol} / \mathrm{L})\end{array}$ & $80.77 \pm 12.4$ & $87.44 \pm 81.8$ & 0.004 & $63.44 \pm 12$ & $68.59 \pm 25.08$ & $<0.001$ \\
\hline Urea (mmol/L) & $5.1 \pm 1.23$ & $6 \pm 3.24$ & $<0.001$ & $4.51 \pm 1.23$ & $5.45 \pm 1.98$ & $<0.001$ \\
\hline $\mathrm{UA}(\mathrm{mmol} / \mathrm{L})$ & $365.08 \pm 89.14$ & $319.21 \pm 100.07$ & 0.159 & $270.07 \pm 62.98$ & $286.69 \pm 95.89$ & $<0.001$ \\
\hline eGFR & $94.17 \pm 13.18$ & $90.39 \pm 18.27$ & 0.001 & $99.36 \pm 14.61$ & $88.3 \pm 20.48$ & $<0.001$ \\
\hline IRON ( $\mu \mathrm{mol} / \mathrm{L})$ & $23.35 \pm 8.46$ & $14.14 \pm 6.4$ & 0.001 & $17.8 \pm 6.59$ & $13.55 \pm 5.91$ & 0.283 \\
\hline $\begin{array}{l}\text { Ferritin } \\
\text { (ng/mL) }\end{array}$ & $206.22 \pm 191.69$ & $274.03 \pm 191.58$ & $<0.001$ & $92.01 \pm 79.14$ & $233.5 \pm 178.27$ & $<0.001$ \\
\hline $\mathrm{UIBC}(\mu \mathrm{mol} / \mathrm{L})$ & $33.78 \pm 10.15$ & $34.38 \pm 7.57$ & 0.103 & $42.29 \pm 12.83$ & $34.02 \pm 7.25$ & $<0.001$ \\
\hline $\mathrm{TIBC}(\mu \mathrm{mol} / \mathrm{L})$ & $57.14 \pm 8.13$ & $48.52 \pm 8.9$ & $<0.001$ & $60.09 \pm 11.04$ & $47.56 \pm 8.42$ & 0.002 \\
\hline $\begin{array}{l}\text { Tfs } \\
\text { (transferrin } \\
\text { saturation, \%) }\end{array}$ & $41.15 \pm 14.23$ & $28.7 \pm 11.33$ & 0.009 & $30.5 \pm 11.77$ & $28.12 \pm 10.55$ & 0.456 \\
\hline $\begin{array}{l}\text { Iron deficiency } \\
(\%)\end{array}$ & 0 & $65(18.6)$ & & 0 & $83(28.2)$ & $<0.001$ \\
\hline $\begin{array}{l}\text { Hypertension } \\
\mathrm{n}(\%)\end{array}$ & & 132(37.82) & & & 108(36.73) & 0.77 \\
\hline $\begin{array}{l}\text { Clopidogrel n } \\
(\%)\end{array}$ & & $6(4.55)$ & & & $5(4.63)$ & 0.97 \\
\hline $\begin{array}{l}\text { ACE inhibitors } \\
\mathrm{n}(\%)\end{array}$ & & 10(7.58) & & & $1(0.93)$ & $<0.001$ \\
\hline ARB n (\%) & & $33(25)$ & & & $29(26.85)$ & 0.74 \\
\hline $\begin{array}{l}\beta \text {-blockers n } \\
(\%)\end{array}$ & & $28(21.21)$ & & & $20(18.52)$ & 0.6 \\
\hline Calcium channe & blockers n (\%) & $36(27.27)$ & & & $30(27.78)$ & 0.97 \\
\hline Diuretics n (\%) & & $9(6.82)$ & & & 10(9.26) & 0.48 \\
\hline Diabetes n (\%) & & 113(32.38) & & & $85(28.91)$ & 0.34 \\
\hline \multicolumn{7}{|c|}{ Diabetes management } \\
\hline Lifestyle modific & ation n (\%) & $15(13.27)$ & & & 10(11.76) & $<0.001$ \\
\hline Oral agents only & $n(\%)$ & $33(29.2)$ & & & $35(41.18)$ & $<0.001$ \\
\hline $\begin{array}{l}\text { Insulin only n } \\
(\%)\end{array}$ & & $64(56.64)$ & & & $30(35.29)$ & $<0.001$ \\
\hline Oral agents and & nsulin n (\%) & $1(0.88)$ & & & 10(11.76) & $<0.001$ \\
\hline Family History o & CAD n (\%) & 106(30.37) & & & $66(22.45)$ & 0.024 \\
\hline
\end{tabular}




\begin{tabular}{|lccc|}
\hline \multicolumn{1}{|c|}{ male } & female & \\
\hline $\begin{array}{l}\text { Dislipidemia } \mathrm{n} \\
(\%)\end{array}$ & $130(37.25)$ & $72(24.49)$ & $<0.001$ \\
\hline $\begin{array}{l}\text { Statins } \mathrm{n}(\%) \\
\begin{array}{l}\text { Rehospitalized } \\
\text { ratio }\end{array}\end{array}$ & $75(57.69)$ & $42(58.33)$ & 0.93 \\
\hline
\end{tabular}

\section{Correlation between ferritin and other metabolic variables in CAD patients}

We analyzed the correlation between ferritin and other metabolic variables in CAD by Pearson correlation. We totally tested the correlation between serum ferritin and 17 biomarkers, i.e. blood lipids (LDL-C, HDL-C, TC, TG, $A p o A, A p o B$ and $L p(a)$ ), blood glucose (FPG, HbA1c), blood pressure (systolic blood pressure, SBP and diastolic blood pressure, DBP), eGFR, proinflammatory measures (hs-CRP), adiposity measure (BMI) and other iron metabolism markers. TC, TG, LDL-C and Tfs were positively correlated with ferritin in men, HDL-C, ApoA, and eGFR were negatively correlated with ferritin in women, while BMI was inversely associated with ferritin in women (Table 2). UIBC and TIBC were negatively correlated with ferritin in both sexes. Interestingly, $\mathrm{HbA} 1 \mathrm{C}$ level was positively correlated with ferritin in men, while inversely associated with ferritin in women. 
Table 2

Correlation between ferritin and other metabolic variables in CAD patients

\begin{tabular}{|c|c|c|c|c|}
\hline \multirow[b]{2}{*}{ variables } & \multicolumn{2}{|c|}{ male $(n=349)$} & \multicolumn{2}{|c|}{ female $(n=294)$} \\
\hline & $r$ & $P$ & $r$ & $P$ \\
\hline eGFR & 0.023 & 0.706 & -0.267 & $<0.001$ \\
\hline \multicolumn{5}{|l|}{ Blood lipids } \\
\hline $\mathrm{TC}(\mathrm{mmol} / \mathrm{L})$ & 0.131 & 0.003 & -0.075 & 0.154 \\
\hline TG $(\mathrm{mmol} / \mathrm{L})$ & 0.198 & $<0.001$ & 0.085 & 0.105 \\
\hline $\mathrm{HDL}(\mathrm{mmol} / \mathrm{L})$ & -0.033 & 0.450 & -0.196 & $<0.001$ \\
\hline $\mathrm{LDL}(\mathrm{mmol} / \mathrm{L})$ & 0.107 & 0.014 & -0.059 & 0.263 \\
\hline $\mathrm{APOA}(\mathrm{g} / \mathrm{L})$ & -0.066 & 0.254 & -0.168 & 0.008 \\
\hline APOB (g/L) & 0.053 & 0.360 & 0.077 & 0.222 \\
\hline Lpa $(g / L)$ & -0.032 & 0.584 & 0.078 & 0.195 \\
\hline \multicolumn{5}{|l|}{ Blood glucose } \\
\hline $\mathrm{HbA1C}(\%)$ & -0.085 & 0.041 & 0.188 & $<0.001$ \\
\hline \multicolumn{5}{|l|}{ Blood pressure } \\
\hline $\mathrm{SBP}(\mathrm{mmHg})$ & 0.069 & 0.095 & 0.069 & 0.167 \\
\hline $\mathrm{DBP}(\mathrm{mmHg})$ & 0.029 & 0.486 & 0.059 & 0.234 \\
\hline \multicolumn{5}{|l|}{ Inflammation } \\
\hline $\mathrm{hsCRP}(\mathrm{mg} / \mathrm{L})$ & 0.049 & 0.541 & -0.089 & 0.341 \\
\hline \multicolumn{5}{|l|}{ Adiposity } \\
\hline BMI (m/kg2) & 0.041 & 0.324 & 0.115 & 0.02 \\
\hline \multicolumn{5}{|l|}{ Iron metabolic markers } \\
\hline IRON $(\mu \mathrm{mol} / \mathrm{L})$ & .081 & 0.051 & -0.216 & $<0.001$ \\
\hline $\mathrm{UIBC}(\mu \mathrm{mol} / \mathrm{L})$ & -0.262 & $<0.001$ & -0.295 & $<0.001$ \\
\hline $\operatorname{TIBC}(\mu \mathrm{mol} / \mathrm{L})$ & -0.168 & $<0.001$ & -0.393 & $<0.001$ \\
\hline Tfs (transferrin saturation, \%) & 0.128 & 0.002 & -0.096 & 0.054 \\
\hline \multicolumn{5}{|c|}{ Bold values indicate significant results with $P<0.05$. } \\
\hline \multicolumn{5}{|c|}{$\begin{array}{l}\text { eGFR: estimated glomerular filtration rate, BMI: body mass index, HbA1c: glycosylated haemoglobin, TC: tota } \\
\text { cholesterol, LDL-C: low-density lipoprotein cholesterol, HDL-C: high-density lipoprotein cholesterol. }\end{array}$} \\
\hline \multicolumn{5}{|c|}{$\begin{array}{l}\text { lipoprotein cholesterol, TG triglycerides, SBP systolic blood pressure, DBP: diastolic blood pressure, hs-CRP: } \\
\text { high sensitive C-reactive protein. }\end{array}$} \\
\hline \multicolumn{5}{|c|}{$\begin{array}{l}\text { Pearson correlation was performed with ferritin and other metabolic variables in CAD patients. Continuous } \\
\text { variables with skewed distributions (ferritin, } T G \text {,hs-CRP) were log transformed for analysis. }\end{array}$} \\
\hline
\end{tabular}




\section{Logistic regression analysis of iron metabolic markers and the risk of CAD and 1-year rehospitalization}

To illustrate the continuous relationships between parameters of iron metabolism and the risk of CAD, we assessed the concentration-risk relationship between serum UIBC, ferritin levels and CAD risk by multivariate random-effects meta-regression based on the restricted cubic spline model with four knots (Figure 2). Visual inspection revealed Jshaped relationships between UIBC, ferritin and the ORs for the risk of CAD. The association between ferritin and high CAD was further explored by categorizing ferritin levels into low, normal and high groups, and using the normal ferritin level as the reference.

From an unadjusted multivariable logistic regression analysis, each unit increase in ferritin was associated with a 1.003-fold ( $95 \%$ confidence interval $(\mathrm{Cl}), 1.002-1.004)$ and 1.01 -fold ( $95 \% \mathrm{Cl}, 1.000-1.01)$ increased Odds Ratio (OR) of CAD in male and female groups respectively (Table 3). Serum iron level and Tfs were found to have decreased OR for CAD in both sexes (iron OR=0.83, 95\% $\mathrm{Cl} 0.803-0.858$ in men, OR=0.894, 95\% Cl 0.860-0.929 in women, Tfs $\mathrm{OR}=0.896,95 \% \mathrm{Cl} 0.867-0.925$ in men, $\mathrm{OR}=0.977,95 \% \mathrm{Cl} 0.957-0.997$ in women, $P<0.05$, Table 3). Patients with low serum level of UIBC, TIBC, and Iron deficiency had significantly lower risks for $C A D$ in women $(O R=0.214,95 \% \mathrm{Cl}$ (0.145-0.315), $P<0.001$, Table 3). 
Table 3

Associations of iron metabolic markers and CAD

\begin{tabular}{|c|c|c|c|c|}
\hline \multirow[t]{2}{*}{ Models } & \multicolumn{2}{|c|}{ male (Control/CAD 381/349) } & \multicolumn{2}{|c|}{ Female (Control/CAD 262/294) } \\
\hline & OR $(95 \% \mathrm{Cl})$ & $P$ & OR $(95 \% \mathrm{Cl})$ & $P$ \\
\hline \multicolumn{5}{|l|}{ Model 1} \\
\hline Iron ( $\mu \mathrm{mol} / \mathrm{L})$ & $0.83(0.803-0.858)$ & $<0.001$ & $0.894(0.860-0.929)$ & $<0.001$ \\
\hline Ferritin (ng/mL) & $1.003(1.002-1.004)$ & $<0.001$ & $1.01(1-1.01)$ & 0.017 \\
\hline $\mathrm{UIBC}(\mu \mathrm{mol} / \mathrm{L})$ & $1.008(0.989-1.027)$ & 0.417 & $0.88(0.79-0.98)$ & 0.018 \\
\hline $\operatorname{TIBC}(\mu \mathrm{mol} / \mathrm{L})$ & $0.961(0.825-1.12)$ & 0.613 & $0.89(0.86-0.92)$ & $<0.001$ \\
\hline Normal ferritin & 1 (Reference) & & & \\
\hline low ferritin & $0.466(0.306-0.709)$ & $<0.001$ & $0.2(0.1-0.4)$ & $<0.001$ \\
\hline high ferritin & $0.371(0.102-1.353)$ & 0.133 & $0.12(0.03-0.42)$ & 0.001 \\
\hline Tfs (transferrin saturation, \%) & $0.89(0.869-0.911)$ & $<0.001$ & $0.977(0.957-0.997)$ & 0.022 \\
\hline Iron deficiency & $1.203(0.77-1.881)$ & 0.417 & $0.214(0.145-0.315)$ & $<0.001$ \\
\hline \multicolumn{5}{|l|}{ Model 2} \\
\hline Iron $(\mu \mathrm{mol} / \mathrm{L})$ & $0.841(0.805-0.878)$ & $<0.001$ & $0.888(0.847-0.931)$ & $<0.001$ \\
\hline Ferritin $(\mathrm{ng} / \mathrm{mL})$ & $1.005(1.003-1.007)$ & $<0.001$ & $1.010(1.007-1.013)$ & $<0.001$ \\
\hline normal ferritin & 1 (Reference) & & 1 (Reference) & \\
\hline low ferritin & $0.297(0.175-0.504)$ & $<0.001$ & $0.265(0.111-0.635)$ & 0.003 \\
\hline high ferritin & $0.168(0.032-1.072)$ & 0.092 & $0.269(0.048-1.503)$ & 0.135 \\
\hline Tfs (transferrin saturation, \%) & $0.929(0.909-0.949)$ & $<0.001$ & $0.862(0.82-0.905)$ & $<0.001$ \\
\hline $\mathrm{UIBC}(\mu \mathrm{mol} / \mathrm{L})$ & / & / & $0.824(0.775-0.876)$ & $<0.001$ \\
\hline $\mathrm{TIBC}(\mu \mathrm{mol} / \mathrm{L})$ & l & I & $0.873(0.840-0.907)$ & $<0.001$ \\
\hline Iron deficiency & / & / & $8.905(0.635-124.873)$ & 0.105 \\
\hline \multicolumn{5}{|l|}{ Model 3} \\
\hline Iron $(\mu \mathrm{mol} / \mathrm{L})$ & $0.889(0.626-1.264)$ & 0.513 & $0.806(0.687-0.944)$ & 0.008 \\
\hline Ferritin $(\mathrm{ng} / \mathrm{mL})$ & $1.029(1.002-1.058)$ & 0.037 & $1.02(1.005-1.034)$ & 0.008 \\
\hline normal ferritin & 1 (Reference) & & 1 (Reference) & \\
\hline low ferritin & $39.138(0.941-162.564)$ & 0.054 & $0.087(0.01-0.724)$ & 0.024 \\
\hline high ferritin & l & l & $0.072(0.005-0.999)$ & 0.05 \\
\hline Tfs (transferrin saturation, \%) & $0.87(0.695-1.089)$ & 0.223 & $0.977(0.896-1.066)$ & 0.599 \\
\hline UIBC $(\mu \mathrm{mol} / \mathrm{L})$ & I & I & $0.919(0.852-0.992)$ & 0.031 \\
\hline
\end{tabular}




\begin{tabular}{|llll|}
\hline Models & \multicolumn{2}{c|}{ male (Control/CAD 381/349) } & \multicolumn{2}{l|}{ Female (Control/CAD 262/294) } \\
\cline { 2 - 4 } & \multicolumn{1}{c|}{ OR $(95 \% \mathrm{Cl})$} & OR $(95 \% \mathrm{Cl})$ & $P$ \\
\hline TIBC $(\mu \mathrm{mol} / \mathrm{L})$ & $/$ & $0.864(0.787-0.95)$ & 0.002 \\
\hline Model 1 is crude model without any confounders & & \\
\hline $\begin{array}{l}\text { Model } 2 \text { was adjusted for age, BMl, SBP, DBP, Heart ratio, smoking habit, alcohol drinking habit, hypertension, } \\
\text { diabetes status, dyslipidemia, medicine and family history of CAD. }\end{array}$ & \\
\hline \multicolumn{2}{l}{ Model 3 additionally adjusted for HbA1C, ALT, AST, TCHO, TG, hs-CRP, eGFR. } \\
\hline
\end{tabular}

When further adjusted for age, BMI, SBP, DBP, HR, smoking habit, alcohol drinking habit, hypertension, diabetes status, dyslipidemia, medicine and family history of CAD (model 2), the effect modification by serum iron, ferritin levels and Tfs on the CAD risk remained similar significant in both sexes. Additional adjustment for laboratory tests including HbA1C, ALT, AST, TCHO, TG, hs-CRP, eGFR (model 3) there was no significant direct correlation for serum iron and Tfs on the CAD risk in men ( $P>0.05)$. Serum level of iron (OR=0.806, 95\% $\mathrm{Cl}(0.687-0.944), \mathrm{UIBC}$ $\mathrm{OR}=0.919,95 \% \mathrm{Cl}(0.852-0.992)$, and TIBC $(\mathrm{OR}=0.864,95 \% \mathrm{Cl}(0.787-0.95)$ were found to have a protective role for CAD in women $(P<0.05$, Table 3). The OR for ferritin was significant in the both sexes (OR $=1.029,95 \% \mathrm{Cl}(1.002-$ 1.058 ) in men, $\mathrm{OR}=1.02,95 \% \mathrm{Cl}(1.005-1.034)$ in women, $P<0.05$, Table 3$)$. When analyzed with the normal ferritin group as the reference, the low level of serum ferritin showed a decreased OR for CAD in women $(O R=0.087,95 \% \mathrm{Cl}$ (0.01-0.724) $P<0.05$, Table 3).

Regrettably, there was no statistical significance between the correlation of iron metabolism markers and 1-year rehospitalization after calibrating for confounders (model $3, P>0.05$, Table 4). 
Table 4

Associations of iron metabolic markers and 1-year rehospitalization after coronary revascularization

\begin{tabular}{|c|c|c|c|c|}
\hline \multirow[t]{2}{*}{ Models } & \multicolumn{2}{|c|}{ male (Control/CAD 381/349) } & \multicolumn{2}{|c|}{ female(Control/CAD 262/294) } \\
\hline & OR (95\% Cl) & $P$ & OR $(95 \% \mathrm{Cl})$ & $P$ \\
\hline \multicolumn{5}{|l|}{ Model 1} \\
\hline Iron $(\mu \mathrm{mol} / \mathrm{L})$ & $0.944(0.917-0.973)$ & $<0.001$ & $0.8(0.64-1.01)$ & 0.059 \\
\hline Ferritin (ng/mL) & $1.001(1-1.003)$ & 0.026 & $1(0.996-1.01)$ & 0.276 \\
\hline $\mathrm{UIBC}(\mu \mathrm{mol} / \mathrm{L})$ & $0.997(0.973-1.022)$ & 0.839 & $1.04(0.96-1.13)$ & 0.369 \\
\hline $\mathrm{TIBC}(\mu \mathrm{mol} / \mathrm{L})$ & $0.961(0.939-0.983)$ & 0.001 & $0.96(0.94-0.99)$ & 0.01 \\
\hline Normal ferritin & 1 (Reference) & & 1 (Reference) & \\
\hline low ferritin & $0.677(0.429-1.071)$ & 0.095 & $0.45(0.19-1.06)$ & 0.068 \\
\hline high ferritin & $0.75(0.152-3.695)$ & 0.724 & $0.05(0-0.59)$ & 0.018 \\
\hline Tfs (transferrin saturation, \%) & $0.977(0.961-0.994)$ & 0.008 & $0.977(0.961-0.994)$ & 0.155 \\
\hline Iron deficiency & $0.882(0.509-1.53)$ & 0.665 & $0.882(0.509-1.53)$ & 0.67 \\
\hline \multicolumn{5}{|l|}{ Model 2} \\
\hline $\operatorname{Iron}(\mu \mathrm{mol} / \mathrm{L})$ & $0.95(0.92-0.981)$ & 0.002 & $0.96(0.91-1)$ & 0.052 \\
\hline Ferritin (ng/mL) & $1.001(1-1.003)$ & 0.027 & $1.001(0.999-1.002)$ & 0.526 \\
\hline normal ferritin & 1 (Reference) & & 1 (Reference) & \\
\hline low ferritin & l & / & $0.81(0.46-1.43)$ & 0.462 \\
\hline high ferritin & I & I & $0.2(0.02-1.89)$ & 0.161 \\
\hline Tfs (transferrin saturation, \%) & $0.98(0.963-0.998)$ & 0.032 & / & / \\
\hline $\mathrm{UIBC}(\mu \mathrm{mol} / \mathrm{L})$ & / & / & $0.99(0.96-1.03)$ & 0.662 \\
\hline $\mathrm{TIBC}(\mu \mathrm{mol} / \mathrm{L})$ & $0.967(0.943-0.991)$ & 0.007 & $0.971(0.944-0.999)$ & 0.043 \\
\hline Iron deficiency & l & / & $0.982(0.959-1.006)$ & 0.141 \\
\hline \multicolumn{5}{|l|}{ Model 3} \\
\hline Iron $(\mu \mathrm{mol} / \mathrm{L})$ & $0.972(0.916-1.031)$ & 0.345 & $1.032(0.924-1.154)$ & 0.574 \\
\hline Ferritin (ng/mL) & $1.001(0.998-1.003)$ & 0.576 & l & / \\
\hline Tfs (transferrin saturation, \%) & $0.979(0.945-1.013)$ & 0.226 & / & / \\
\hline $\operatorname{TIBC}(\mu \mathrm{mol} / \mathrm{L})$ & $1.009(0.959-1.062)$ & 0.733 & $1.015(0.947-1.088)$ & 0.674 \\
\hline \multicolumn{5}{|c|}{ Model 1 is crude model without any confounders } \\
\hline
\end{tabular}




\section{OR $(95 \% \mathrm{Cl})$}

$P$

OR $(95 \% \mathrm{Cl})$

$P$

Model 3 additionally adjusted for HbA1C, ALT, AST, TC, TG, hs-CRP, eGFR.

\section{Association of body iron status with the severity of CAD}

The severity of CAD was quantified the modified Gensini scores based on the number and the extent of lesions in coronary arteries [32]. The detail calculation of modified Gensini scores was described in our previous study[33]. Serum iron status markers exhibited no significant association with the severity of CAD (Supplement Table 1).

\section{Discussion}

There are limited data concerning serum iron and iron saturation in CAD patients and the results are also inconsistent. This study was conducted to assess the association between serum iron metabolism markers and CAD. For physiological reasons, the reference interval for iron metabolism markers in women are different from men, we analyzed the association of iron status and CAD stratified by gender. In a cohort of 643 CAD patients and 643 controls, iron imbalance, as characterized by either high serum ferritin or low iron levels, was associated with an increased risk of CAD.

Overall, our findings suggested that low Serum level of iron, UIBC, TIBC and ferritin levels showed a protective role for CAD in women, but not in men. Increased serum ferritin was independently associated with CAD in men and women. Our data add to previous reports that showed a protective role of low iron level on the risk of CAD. Sullivan proposed that reduced iron stores can protect against ischemic heart disease and for the first time to explain the sex difference in CAD risk [34]. Salonen, J.T. et al. observed that a higher level of iron is a risk factor for myocardial infarction in Finnish men [35]. Later, it has been reported that reducing iron stores through phlebotomy could decelerate the progression of atherosclerotic plaque $[12,22]$. However, we did not find iron deficiency was associated with increased risk for CAD, which was inconsistent with some previous report. Iron deficiency was associated with increased risk for CAD and had detrimental effects in patients with CAD [1,26]. The patients' status and comorbidities might explain the inconsistence. In our study, we enrolled low percentage (less than $30 \%$ ) of iron deficiency in the patient group, and we exclude the CAD patients with clinical anemia. However, there was a high prevalence of iron deficiency in acute coronary syndrome and its association with poor outcome [36]. Moreover, the definition of iron deficiency to be applied in heart disease remains controversial.

Iron is a trace element that exists in serum at low concentration of $\mathrm{mg} / \mathrm{dL}$. Iron values exhibit diurnal variation depending on dietary iron intake or patient condition [37]. The iron measurements may be affected by recent food ingestion. Various previous studies have evaluated serum ferritin instead of serum iron level. Ferritin is an ironbinding molecule that stores iron in a biologically available form, which is essential to iron homeostasis [38]. Moreover, serum ferritin is a well-known acute-phase reactant. Nevertheless, there are inconsistent results concerning the relation of serum ferritin with CAD. A batch of studies have shown a similar positive correlation between ferritin and the risk of CAD [7, 17, 19, 20, 23, 24, 39-41]. Elevated serum ferritin was independently significantly associated with carotid atherosclerosis in women (Xu et al., 2017). Consistent with previous positive results, in our study, we found serum ferritin was independently significantly associated with CAD in both sexes. On the other hand, some other studies demonstrated that the higher risk of CAD is not related to the serum ferritin 
levels $[24,42]$. These studies implied that ferritin might be an inflammatory marker for atherosclerosis. It is hard to tell whether high ferritin levels reflect inflammation caused by hyperglycemia or indicate iron overload, which can also lead to inflammation.

Iron is an essential mineral, which participates in different functions of the organism under physiological conditions. Numerous biological processes, such as oxygen and lipid metabolism, protein production, cellular respiration, and DNA synthesis, require the presence of iron [43]. Maintaining iron metabolism is important for cardiovascular health as its high energy consumption and high mitochondrial activity [44]. Intravenous iron administration in acute myocardial infarction (MI) exerted beneficial effects in MI patients [45]. However, excessive iron accumulation accelerates the formation of atherosclerosis through several putative mechanisms. Firstly, iron catalyzes Fenton reaction to produce reactive oxygen species (ROS) which promote LDL peroxidation and induce endothelial dysfunction by reducing the bioavailability of nitric oxide [46]. Secondly, excess iron may make vascular endothelium vulnerable to other pathogenic factors. Ultimately, atherosclerosis is accelerated as a result of increased platelet activity and leucocyte adhesion [47]. Thirdly, ROS increased the expression of LOX-1 receptor on endothelial cells, resulting in mitochondrial DNA damage and autophagy activation [48]. Fourthly, accumulated iron in adipocytes leads to adipocyte IR by increasing lipolysis and by decreasing insulin-stimulated glucose transport [2]. Adipocyte IR accelerates atherosclerosis [49].

\section{Conclusion}

There are limited data concerning serum iron and iron saturation in CAD patients and the results are also inconsistent. The discrepancies among the studies may be partly attributable to the differences in race, dietary habits, sample size and confounding factors. Low Serum level of iron, UIBC, TIBC and ferritin levels were found to have a protective role for CAD in women, but not in men. Increased serum ferritin is independently associated with CAD in men and women. The main limitation of the study is its cross-sectional design; therefore, a causal relationship could not be established. In addition, the measurements of hepcidin, a well-known regulator of body iron fluxes, were not available. However, we evaluated transferrin saturation which is an important determinant of hepcidin release. Moreover, the daily iron intake needs to be estimated in the future study. Finally, there is a pragmatic need to identify circulating iron biomarkers reliably characterizing iron status within tissues.

\section{Abbreviations}

UIBC: unsaturated transferrin iron binding capacity; TIBC: Total transferrin iron binding capacity; TC: total cholesterol: TG: Triglyceride; CAG: cardioangiography.SBP: Systolic blood pressure; DBP: Diastolic blood pressure; BMI: Body mass index; LDL-C: low-density lipoprotein cholesterol; HDL-C: high-density lipoprotein cholesterol; hsCRP: High sensitivity $C$ reactive protein; AST: Aspartate aminotransferase; ALT: Alanine aminotransferase; $\gamma^{-G T}{ }^{-}{ }^{-}$ glutamyl transferase; eGFR: Estimated glomerular filtration rate; BP: Blood pressure; Tfs: transferrin saturation; IR: insulin resistance; TC: total cholesterol; UREA: urea nitrogen; UA: uric acid; HbA1c:Hemoglobin A1c; Hcy: homocysteine; ApoA: Apolipoprotein A; ApoB: Apolipoprotein B; TP: Total proteins; ALB: Albumin.

\section{Declarations}

\section{Conflict of Interest}


The authors declare that the research was conducted in the absence of any commercial or financial relationships that could be construed as a potential conflict of interest.

\section{Funding}

The First Affiliated Hospital of Zhengzhou University financially supported this study. The funders had no role in study design, data collection and analysis, decision to publish, or preparation of the manuscript.

\section{Acknowledgments}

Thanks to Liming Liu (Department of Cardiology, The First Affiliated Hospital of Zhengzhou University) for the Guidance in Coronary artery disease.

\section{Authors' contributions}

Each author is expected to have made substantial contributions. Shuren Guo performed the experiments, analyzed the data, prepared figures and tables, and reviewed drafts of the paper. Xiaohuan Mao performed the data collection and analysis, Xiaohua Li, Huan Ouyang and Yuhua Gao performed the laboratory tests and clincal data collection. Liang Ming have drafted the work and substantively revised it. All authors have approved the submitted version and agreed to be accountable for all aspects of work ensuring integrity and accuracy. All authors read and approved the final manuscript.

\section{References}

1. Altamura S, Mudder K, Schlotterer A et al. (2021) Iron aggravates hepatic insulin resistance in the absence of inflammation in a novel db/db mouse model with iron overload. Molecular metabolism 51:101235

2. Cannizzo B, Lujan A, Estrella $\mathrm{N}$ et al. (2012) Insulin resistance promotes early atherosclerosis via increased proinflammatory proteins and oxidative stress in fructose-fed ApoE-KO mice. Experimental diabetes research 2012:941304

3. Cook JD (2005) Diagnosis and management of iron-deficiency anaemia. Best practice \& research. Clinical haematology 18:319-332

4. Danesh J, Appleby P (1999) Coronary heart disease and iron status: meta-analyses of prospective studies. Circulation 99:852-854

5. Diaz-Lopez A, Iglesias-Vazquez L, Palleja-Millan M et al. (2020) Association between Iron Status and Incident Type 2 Diabetes: A Population-Based Cohort Study. Nutrients 12

6. Ding Z, Liu S, Wang X et al. (2014) LOX-1, oxidant stress, mtDNA damage, autophagy, and immune response in atherosclerosis. Canadian journal of physiology and pharmacology 92:524-530

7. Ekblom K, Marklund SL, Jansson JH et al. (2011) Iron stores and HFE genotypes are not related to increased risk of first-time myocardial infarction: a prospective nested case-referent study. International journal of cardiology 150:169-172

8. Florian A, Ludwig A, Rosch S et al. (2014) Positive effect of intravenous iron-oxide administration on left ventricular remodelling in patients with acute ST-elevation myocardial infarction - a cardiovascular magnetic resonance (CMR) study. International journal of cardiology 173:184-189 
9. Guo S, Mao X, Li X et al. (2021) Serum Complement C1q Activity Is Associated With Obstructive Coronary Artery Disease. Frontiers in cardiovascular medicine 8:618173

10. Habib A, Finn AV (2014) The role of iron metabolism as a mediator of macrophage inflammation and lipid handling in atherosclerosis. Frontiers in pharmacology 5:195

11. Haidari M, Javadi E, Sanati A et al. (2001) Association of increased ferritin with premature coronary stenosis in men. Clinical chemistry 47:1666-1672

12. Henjum S, Groufh-Jacobsen S, Stea TH et al. (2021) Iron Status of Vegans, Vegetarians and Pescatarians in Norway. Biomolecules 11

13. Holay MP, Choudhary AA, Suryawanshi SD (2012) Serum ferritin-a novel risk factor in acute myocardial infarction. Indian heart journal 64:173-177

14. Jankowska EA, Rozentryt P, Witkowska A et al. (2010) Iron deficiency: an ominous sign in patients with systolic chronic heart failure. European heart journal 31:1872-1880

15. Kang P, Liu T, Tian C et al. (2012) Association of total iron binding capacity with coronary artery disease. Clinica chimica acta; international journal of clinical chemistry 413:1424-1429

16. Knovich MA, Storey JA, Coffman LG et al. (2009) Ferritin for the clinician. Blood reviews 23:95-104

17. Kruszewski M (2004) The role of labile iron pool in cardiovascular diseases. Acta biochimica Polonica $51: 471-480$

18. Lapice E, Masulli M, Vaccaro O (2013) Iron deficiency and cardiovascular disease: an updated review of the evidence. Current atherosclerosis reports 15:358

19. Levey AS, Stevens LA, Schmid CH et al. (2009) A new equation to estimate glomerular filtration rate. Annals of internal medicine 150:604-612

20. Ma H, Lin H, Hu Y et al. (2015) Serum ferritin levels are associated with carotid atherosclerosis in Chinese postmenopausal women: the Shanghai Changfeng Study. The British journal of nutrition 114:1064-1071

21. Minqin R, Rajendran R, Pan N et al. (2005) The iron chelator desferrioxamine inhibits atherosclerotic lesion development and decreases lesion iron concentrations in the cholesterol-fed rabbit. Free radical biology \& medicine 38:1206-1211

22. Munoz-Bravo C, Gutierrez-Bedmar M, Gomez-Aracena J et al. (2013) Iron: protector or risk factor for cardiovascular disease? Still controversial. Nutrients 5:2384-2404

23. Murphy CJ, Oudit GY (2010) Iron-overload cardiomyopathy: pathophysiology, diagnosis, and treatment. Journal of cardiac failure 16:888-900

24. Peng W, Cai G, Xia Y et al. (2019) Mitochondrial Dysfunction in Atherosclerosis. DNA and cell biology 38:597606

25. Qayyum R, Schulman P (2005) Iron and atherosclerosis. Clinical cardiology 28:119-122

26. Rajapurkar MM, Shah SV, Lele SS et al. (2012) Association of catalytic iron with cardiovascular disease. The American journal of cardiology 109:438-442

27. Rasouli M, Kiasari AM, Arab S (2008) Indicators of dehydration and haemoconcentration are associated with the prevalence and severity of coronary artery disease. Clinical and experimental pharmacology \& physiology 35:889-894

28. Ravingerova T, Kindernay L, Bartekova M et al. (2020) The Molecular Mechanisms of Iron Metabolism and Its Role in Cardiac Dysfunction and Cardioprotection. International journal of molecular sciences 21 
29. Reinhold J, Papadopoulou C, Baral R et al. (2021) Iron deficiency for prognosis in acute coronary syndrome - A systematic review and meta-analysis. International journal of cardiology 328:46-54

30. Reunanen A, Takkunen H, Knekt P et al. (1995) Body iron stores, dietary iron intake and coronary heart disease mortality. Journal of internal medicine 238:223-230

31. Reyes C, Pons NA, Renones CR et al. (2020) Association between serum ferritin and acute coronary heart disease: A population-based cohort study. Atherosclerosis 293:69-74

32. Rodriguez-Mortera R, Caccavello R, Hermo R et al. (2021) Higher Hepcidin Levels in Adolescents with Obesity Are Associated with Metabolic Syndrome Dyslipidemia and Visceral Fat. Antioxidants (Basel) 10

33. Salonen JT, Nyyssonen K, Korpela H et al. (1992) High stored iron levels are associated with excess risk of myocardial infarction in eastern Finnish men. Circulation 86:803-811

34. Sengoelge G, Sunder-Plassmann G, Horl WH (2005) Potential risk for infection and atherosclerosis due to iron therapy. Journal of renal nutrition: the official journal of the Council on Renal Nutrition of the National Kidney Foundation 15:105-110

35. Silvestre OM, Goncalves A, Nadruz W, Jr. et al. (2017) Ferritin levels and risk of heart failure-the Atherosclerosis Risk in Communities Study. European journal of heart failure 19:340-347

36. Steen DL, Cannon CP, Lele SS et al. (2013) Prognostic evaluation of catalytic iron in patients with acute coronary syndromes. Clinical cardiology 36:139-145

37. Sullivan JL (1981) Iron and the sex difference in heart disease risk. Lancet 1:1293-1294

38. Sun Q, Ma J, Rifai N et al. (2008) Excessive body iron stores are not associated with risk of coronary heart disease in women. The Journal of nutrition 138:2436-2441

39. Sung KC, Kang SM, Cho EJ et al. (2012) Ferritin is independently associated with the presence of coronary artery calcium in 12,033 men. Arteriosclerosis, thrombosis, and vascular biology 32:2525-2530

40. Vaquero MP, Martinez-Maqueda D, Gallego-Narbon A et al. (2020) Relationship between iron status markers and insulin resistance: an exploratory study in subjects with excess body weight. PeerJ 8:e9528

41. Von Haehling S, Jankowska EA, Van Veldhuisen DJ et al. (2015) Iron deficiency and cardiovascular disease. Nature reviews. Cardiology 12:659-669

42. Weidmann H, Bannasch JH, Waldeyer C et al. (2020) Iron Metabolism Contributes to Prognosis in Coronary Artery Disease: Prognostic Value of the Soluble Transferrin Receptor Within the AtheroGene Study. Journal of the American Heart Association 9:e015480

43. Weiss G, Goodnough LT (2005) Anemia of chronic disease. The New England journal of medicine 352:10111023

44. Wlazlo N, Van Greevenbroek MM, Ferreira I et al. (2013) Iron metabolism is associated with adipocyte insulin resistance and plasma adiponectin: the Cohort on Diabetes and Atherosclerosis Maastricht (CODAM) study. Diabetes care 36:309-315

45. You SA, Wang Q (2005) Ferritin in atherosclerosis. Clinica chimica acta; international journal of clinical chemistry 357:1-16

46. Yu PH, Lin MY, Chiu YW et al. (2021) Low serum iron is associated with anemia in CKD stage 1-4 patients with normal transferrin saturations. Scientific reports 11:8343

47. Yuan XM, Li W (2003) The iron hypothesis of atherosclerosis and its clinical impact. Annals of medicine 35:578-591

Page $17 / 19$ 
48. Zheng H, Cable R, Spencer B et al. (2005) Iron stores and vascular function in voluntary blood donors. Arteriosclerosis, thrombosis, and vascular biology 25:1577-1583

49. Zhou Y, Liu T, Kang P et al. (2014) Association of better iron status biomarkers and coronary artery disease risk. Internal medicine journal 44:846-850

\section{Figures}

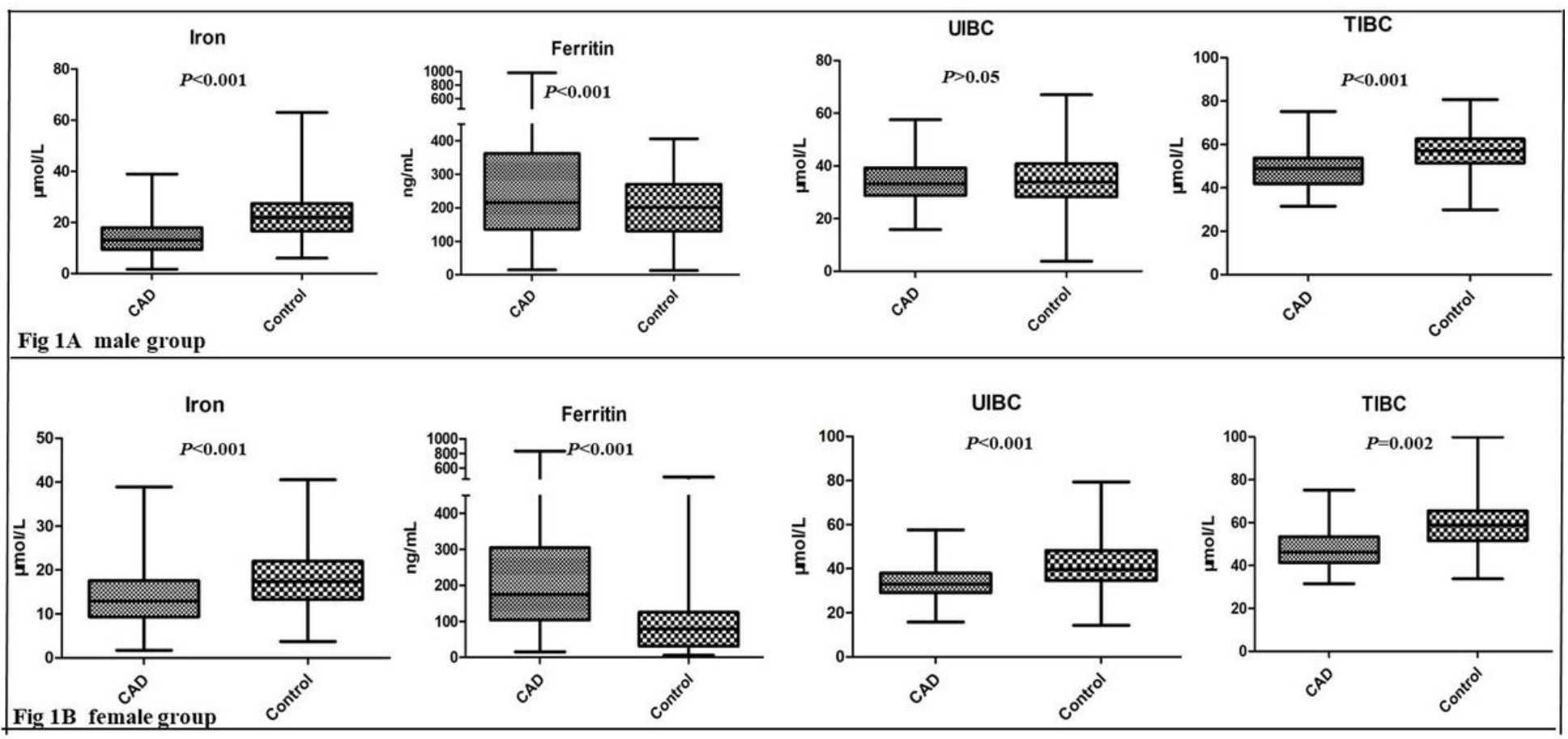

\section{Figure 1}

The serum levels of iron metabolism markers in CAD and Control groups stratified by gender.
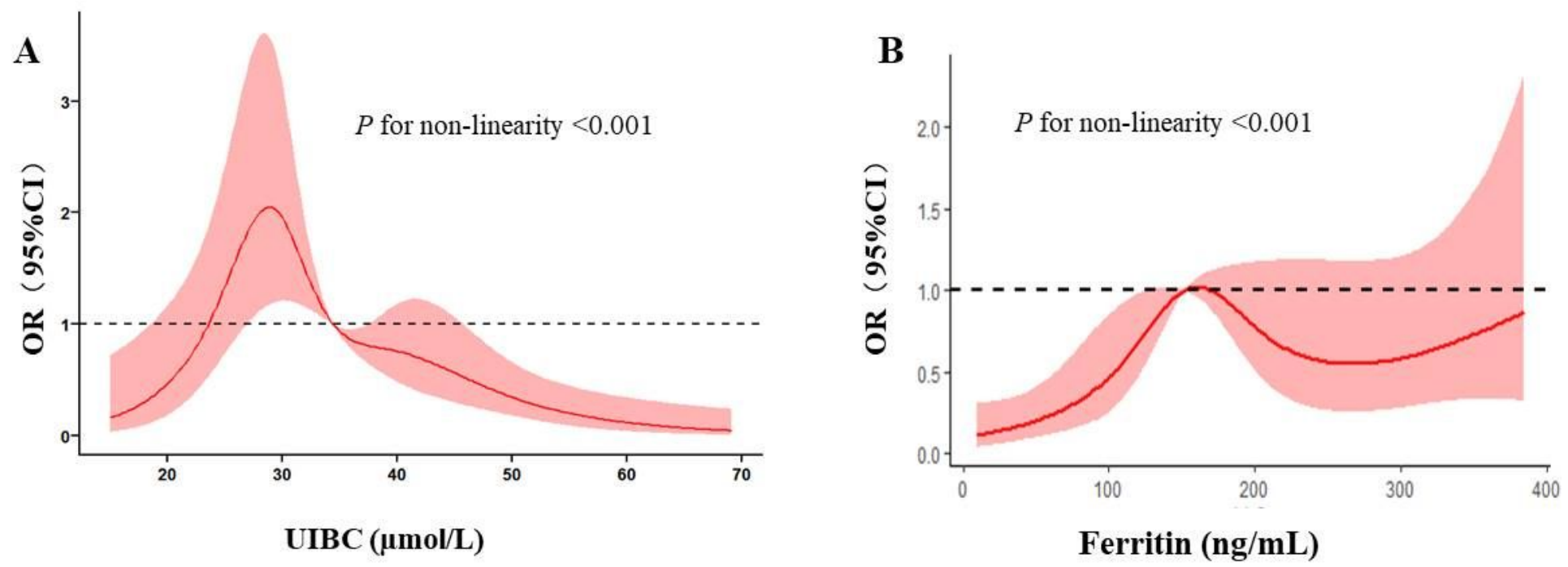

Figure 2 
Concentration-risk relationship between serum $\operatorname{UIBC}(A)$, ferritin levels (B) and risk of CAD. Solid lines show cubic spline interpolation of ORs versus quartile medians, which were all adjusted (model3) for age, sex, BMI, SBP, DBP, $\mathrm{HR}$, smoking habit, alcohol drinking habit, hypertension, diabetes status, dyslipidemia, medicine and family history of CAD, HbA1C, ALT, AST, TC, TG, hs-CRP and eGFR.

\section{Supplementary Files}

This is a list of supplementary files associated with this preprint. Click to download.

- SupplementTable1.docx 